\title{
Acute encephalopathy with elevated CSF inflammatory markers as the initial presentation of COVID-19
}

Shelli Farhadian ${ }^{1,2^{*}}$ (D), Laura R. Glick ${ }^{3}$, Chantal B. F. Vogels ${ }^{4}$, Jared Thomas ${ }^{3}$, Jennifer Chiarella ${ }^{2}$, Arnau Casanovas-Massana ${ }^{4}$, Jing Z Zhou ${ }^{5}$, Camila Odio ${ }^{3}$, Pavithra Vijayakumar ${ }^{3}$, Bertie Geng $^{3}$, John Fournier ${ }^{1}$, Santos Bermejo ${ }^{6}$, Joseph R. Fauver ${ }^{4}$, Tara Alpert ${ }^{4}$, Anne L. Wyllie ${ }^{4}$, Cynthia Turcotte ${ }^{5}$, Matthew Steinle ${ }^{5}$, Patrick Paczkowski ${ }^{5}$, Charles Dela Cruz ${ }^{6}$, Craig Wilen ${ }^{7}$, Albert I. Ko ${ }^{1,4}$, Sean MacKay ${ }^{5}$, Nathan D. Grubaugh ${ }^{4}$, Serena Spudich ${ }^{2}$ and Lydia Aoun Barakat ${ }^{1}$

\begin{abstract}
Background: COVID-19 is caused by the severe acute respiratory syndrome virus SARS-CoV-2. It is widely recognized as a respiratory pathogen, but neurologic complications can be the presenting manifestation in a subset of infected patients.

Case presentation: We describe a 78-year old immunocompromised woman who presented with altered mental status after witnessed seizure-like activity at home. She was found to have SARS-CoV-2 infection and associated neuroinflammation. In this case, we undertake the first detailed analysis of cerebrospinal fluid (CSF) cytokines during COVID-19 infection and find a unique pattern of inflammation in CSF, but no evidence of viral neuroinvasion.

Conclusion: Our findings suggest that neurologic symptoms such as encephalopathy and seizures may be the initial presentation of COVID-19. Central nervous system inflammation may associate with neurologic manifestations of disease.
\end{abstract}

Keywords: COVID-19, SARS-CoV-2, Neuroinflammation

\section{Background}

The novel SARS-CoV-2 coronavirus first emerged in the city of Wuhan, China in December 2019. The virus has since led to the COVID-19 global pandemic, infecting over 2 million people and resulting in over 150,000 deaths to date. SARS-CoV-2 is a Betacoronavirus: the newest of seven strains of coronavirus known to infect humans and the third strain known to cause severe

\footnotetext{
* Correspondence: shelli.farhadian@yale.edu

'Department of Internal Medicine, Section of Infectious Diseases, Yale School of Medicine, New Haven, CT 06510, USA

${ }^{2}$ Department of Neurology, Yale School of Medicine, New Haven, CT 06510, USA

Full list of author information is available at the end of the article
}

disease. While the coronavirus family is widely recognized as respiratory pathogens causing symptoms of upper and lower respiratory tract infection, there are few reported cases of viral invasion into the central nervous system.

Here, we report the clinical presentation and the course of illness of a 78 year-old woman who presented with altered mental status and seizure-like activity and was found to have SARS-CoV-2 associated encephalopathy, with abnormalities in her cerebrospinal fluid (CSF).

\section{Case presentation}

A 78 year-old woman with a history of kidney transplant on immunosuppression presented to our academic

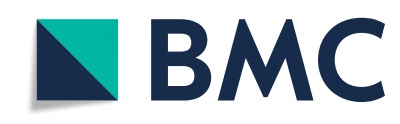

(-) The Author(s). 2020 Open Access This article is licensed under a Creative Commons Attribution 4.0 International License, which permits use, sharing, adaptation, distribution and reproduction in any medium or format, as long as you give appropriate credit to the original author(s) and the source, provide a link to the Creative Commons licence, and indicate if changes were made. The images or other third party material in this article are included in the article's Creative Commons licence, unless indicated otherwise in a credit line to the material. If material is not included in the article's Creative Commons licence and your intended use is not permitted by statutory regulation or exceeds the permitted use, you will need to obtain permission directly from the copyright holder. To view a copy of this licence, visit http://creativecommons.org/licenses/by/4.0/. The Creative Commons Public Domain Dedication waiver (http://creativecommons.org/publicdomain/zero/1.0/) applies to the data made available in this article, unless otherwise stated in a credit line to the data. 
medical center with altered mental status and seizurelike activity in March 2020. The patient was observed at home to have sudden-onset uncontrolled limb movements with ocular deviation followed by several minutes of unresponsiveness. At her baseline, the patient lived independently and had no previous diagnosis of dementia or confusion. However, 3 days prior to admission, she was noted to have confusion and disorientation. In addition, she was noted to have fever and nasal congestion for 2 days prior to admission. The patient was known to be adherent with her medical care and medications including tacrolimus, mycophenolate mofetil, and lisinopril.

Upon arrival the emergency department, the patient was febrile to $100.7 \mathrm{~F}$ but was otherwise hemodynamically stable. On neurological examination, she was noted to be alert and oriented but intermittently confused. She demonstrated bilateral tremor-like movements of the upper and lower extremities without any evidence of convulsions, repetitive movements or rigidity. She had no sensory or motor deficits and her cranial nerve functions were intact. The complete blood count was notable for a pancytopenia with a white blood cell count of $1.810^{3} / \mu \mathrm{L}$, hemoglobin of $10.8 \mathrm{~g} / \mathrm{dL}$, hematocrit of $37.4 \%$ and platelets of $13010^{3} / \mu \mathrm{L}$. Absolute neutrophil count was $0.910^{3} / \mu \mathrm{L}$ and absolute lymphocyte count was $0.610^{3} / \mu \mathrm{L}$. The basic metabolic panel was notable for normal serum electrolytes and stable renal function. Alkaline phosphatase was slightly elevated to $139 \mathrm{U} / \mathrm{L}$ but hepatic function was otherwise unremarkable. Thyroid-stimulating hormone was elevated to $8.1 \mathrm{mIU} /$ L, but thyroxine (T4) was within normal limits. Tacrolimus level was $3.1 \mathrm{mcg} / \mathrm{L}$ (lower than therapeutic range). Procalcitonin was $0.5 \mu \mathrm{g} / \mathrm{L}$ and her respiratory viral panel was negative. Two sets of blood cultures did not grow any organisms. Electrocardiogram showed normal sinus rhythm. Chest X-ray (Fig. 1, left) did not reveal any focal consolidation, pulmonary edema, pleural effusion or pneumothorax.
The patient was admitted to the general medicine floor for further work-up of her altered mental status. She underwent an electroencephalogram (EEG) once at the beginning of her hospital course that demonstrated mild generalized slowing and a magnetic resonance imaging (MRI) scan with contrast that revealed atrophy and patchy periventricular and subcortical white matter hyperintensities, which were interpreted as sequelae of small vessel ischemic disease (Fig. 2).

She subsequently underwent lumbar puncture, which revealed CSF that was clear in appearance with no xanthrochromia. The CSF analysis revealed 350 red cells/uL, 1 white blood cell/uL, 75\% lymphocytes, $25 \%$ monocytes, glucose $67 \mathrm{mg} / \mathrm{dL}$ and protein $43 \mathrm{mg} / \mathrm{dL}$. The CSF herpes simplex virus and varicella zoster virus polymerase chain reactions (PCR) were negative. The plasma cytomegalovirus and adenovirus testing were negative by $\mathrm{PCR}$, and serologies indicated prior Epstein-Barr virus and Parvovirus B19 infection. On hospital day 3, the patient had persistent fevers and developed cough with dyspnea requiring oxygen supplementation. A chest Xray revealed bilateral pulmonary infiltrate (Fig. 1, right). She therefore underwent SARS-CoV-2 PCR testing using a nasopharynx swab and was found to be positive. Sequencing of this SARS-CoV-2 isolate revealed a virus similar to other SARS-CoV-2 circulating in the region (https://nextstrain.org/ncov?s=USA/CT-Yale-009/2020).

Further investigation of the CSF was done following the patient's COVID-19 diagnosis. Inflammatory cytokines were measured in CSF and plasma using a multiplex cytokine assay. This patient's CSF and plasma were tested alongside CSF and plasma specimens obtained from three healthy control volunteers: Female age 63, Female age 45, and Male age 62. (Fig. 3). These control samples were collected during the last year for unrelated studies and were kept frozen until the current cytokine assays, when they were run in parallel with this patient's sample. Levels of Interleukin-6 (IL-6), Interleukin-8 (IL8 ), and Interferon-gamma induced protein-10 (IP-10)
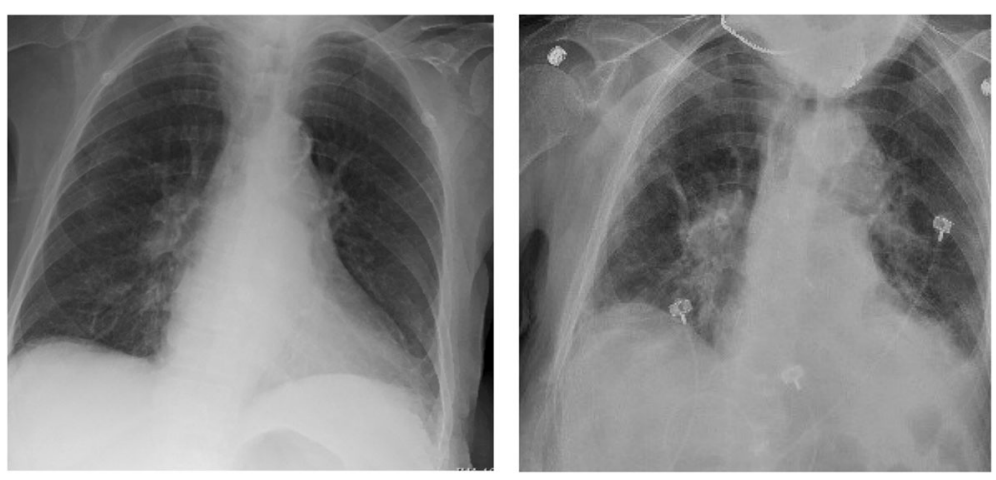

Fig. 1 Chest radiographs. Chest X-ray on admission (left) and on hospital day 13 (right), showing worsening bilateral infiltrates 


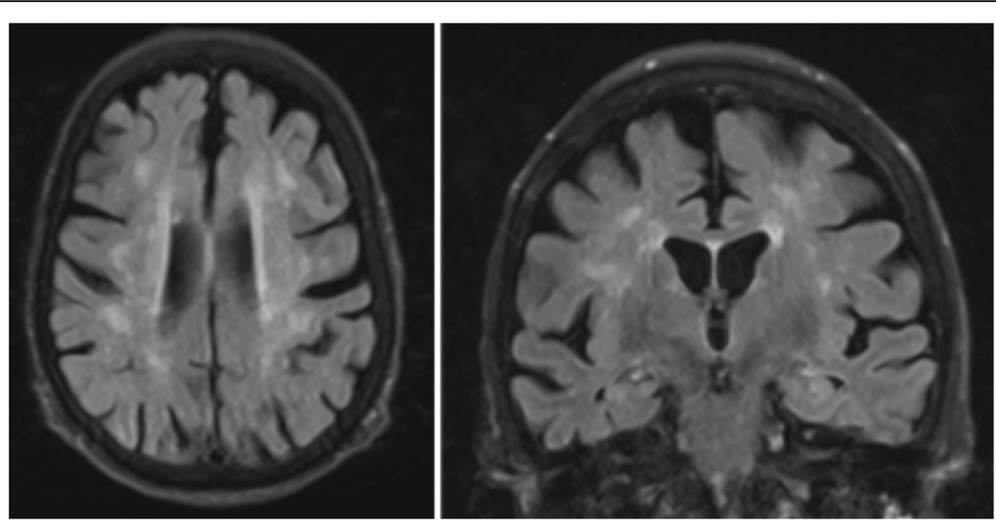

Fig. 2 MRI brain. MRI without contrast demonstrates generalized atrophy and patchy periventricular and subcortical white matter hyperintensities

appeared to be elevated in both CSF and plasma of this patient compared to control, with a unique Monocyte Chemoattractant Protein-1 (MCP-1) signature found only in CSF and not plasma.

The patient's CSF was next tested for the presence of SARS-CoV-2 by several methods and was negative. First, quantitative reverse transcriptase polymerase chain reaction was performed using the Centers for Disease Control SARS-CoV-2 assay. Sequencing of SARS-CoV-2 in the CSF was then attempted using both targeted (tiled PCR-amplicons) and unbiased ("shotgun" metagenomic) approaches. From the targeted sequencing, 316,111 reads were generated from the sample and a total of 60 reads aligned to SARS-CoV-2, which was not above background. From the unbiased sequencing approach, about 40 million reads were generated and none aligned to SARS-CoV-2 or any other respiratory pathogen. Finally, culturing of SARS-CoV-2 was attempted from the CSF supernatant, and after 4 days of culture, SARS-CoV2 by PCR was not detected.

Table 1 summarizes the patient's laboratory findings at the time of diagnosis of COVID-19 and the follow-up course. Based on the patient's age and co-morbidities, she met criteria for treatment initiation per our academic center treatment protocol. The patient received hydroxychloroquine $400 \mathrm{mg}$ twice daily for 1 day followed by $200 \mathrm{mg}$ twice daily for nine additional days. Her confusion slowly improved. However, on hospital day 13, she had worsening of her mental status and became hypoxemic, with chest X-ray showing worsening bilateral infiltrates. She was therefore treated with the IL-6 inhibitor, tocilizumab, with overall improvement in her confusion and respiratory status. The patient was able to be weaned off oxygen and discharged home safely after 1 month of hospitalization. She remained in good condition at the time of this case report.

\section{Discussion and conclusions}

This case highlights the potential for COVID-19 to present with acute neurological symptoms in the absence of respiratory illness. It remains unknown whether central nervous system (CNS) abnormalities observed during COVID-19 are due to viral invasion into the $\mathrm{CNS}$, to damage induced by SARS-CoV-2 induced inflammatory cytokines produced by immune cells within and outside of the CNS, or to a generalized toxic-
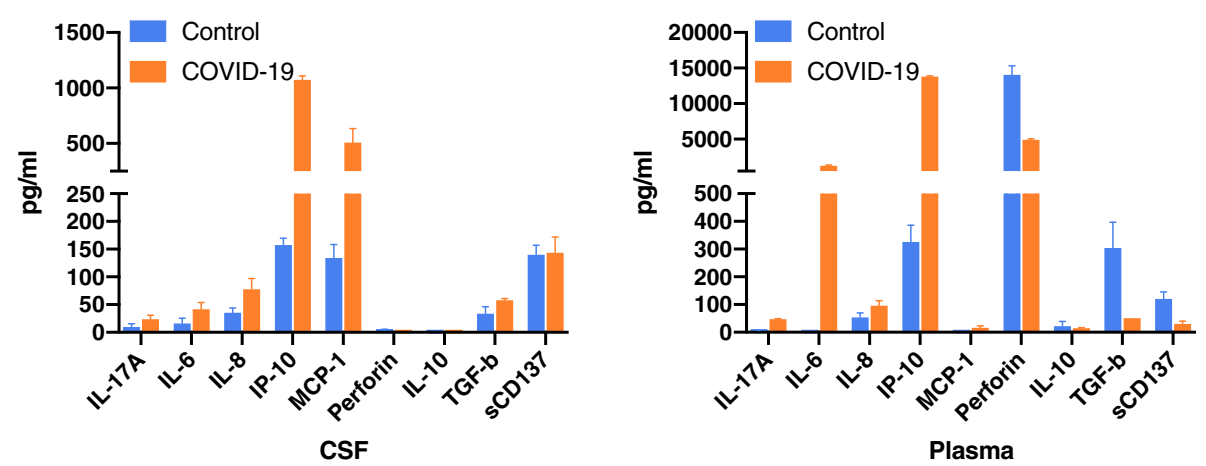

Fig. 3 Cytokine analysis of CSF and plasma. The COVID-19 patient showed a marked increase of a variety of inflammatory cytokine and chemokines in CSF and plasma compare to three control subjects including IL-17A, IL-6, IL-8, IP-10, with a unique MCP-1 signature identified in COVID-19 CSF. All samples were run in duplicate. Mean +/- SD.s 
Table 1 Blood laboratory values throughout the patient's hospital course. Hydroxychloroquine was given day 3 and tocilizumab was given on day 10, as shown

\begin{tabular}{llllllllllllll}
\hline & Day 3 & Day 4 & Day 5 & Day 6 & Day 7 & Day 8 & Day 9 & Day 10 & Day 11 & Day 12 & Day 13 & Day 14 & Day 15 \\
\hline WBC & 0.7 & 2.5 & 7.2 & 11.8 & 7.3 & 5.2 & 3.3 & 5 & 4.5 & 5.1 & 5.1 & 5.3 & 5.4 \\
ALC & - & 0.3 & - & - & 0.5 & & 0.8 & 0.7 & 1.1 & 1.3 & 1.4 & 1.2 & 1.3 \\
CRP & 10.4 & 17.1 & 18.1 & 34.4 & 44.8 & 61.8 & 64.5 & 34.6 & 12.3 & 7.3 & 4.8 & 3.8 & 2.8 \\
Ferritin & 2724 & 3344 & 3214 & 2968 & 2573 & 2512 & 2162 & 2522 & 1801 & 1702 & 1602 & 1389 & 1073 \\
LDH & - & 324 & 333 & 396 & 485 & 381 & 334 & 360 & 298 & 277 & 264 & 269 & 229 \\
Fibrinogen & - & 422 & 405 & - & 423 & 517 & 500 & 498 & 467 & 391 & 413 & 378 & - \\
IL-6 & - & 27.9 & 11 & - & - & - & - & - & - & - & 94 & - \\
\hline
\end{tabular}

Day 3: SARS-CoV-2 positive and start of hydroxychloroquine

Day 10: Tocilizumab given

metabolic encephalopathy associated with critical illness. In this case, we undertook the first detailed analysis of CSF cytokines during COVID-19 infection. We found heightened inflammation in CSF and did not detect SARS-COV-2 virus in the CSF. This patient received experimental therapy with hydroxychloroquine and tocilizumab. Hydroxychloroquine has recently been shown to increase the risk for cardiotoxicity without discernable anti-viral benefit, and it remains unknown whether tocilizumab is effective against COVID-19 [1].

Other coronaviruses (CoVs) have been demonstrated to invade the CNS, infect neurons, and cause an influx of inflammatory cytokines and immune cells in the CNS. Human coronavirus OC43 ( $\mathrm{HCoV}-\mathrm{OC} 43)$ has been shown to infect neurons in vitro, and, in murine models, to invade the CNS causing widespread neuronal damage $[2,3]$. Both SARS and MERS, the two other severe diseases caused by human CoVs, have been associated with CNS manifestations $[4,5]$. In the case of MERS, this was associated with abnormal brain MRI findings of widespread, bilateral hyperintense lesions within the white matter and subcortical areas, and with negative CSF PCR for the MERS-CoV virus. The evidence for neuroinvasion of SARS-CoV-1 is stronger: SARS-CoV-1 has been reported to invade the CNS in mice transgenic for human ACE2 and was detected in human brain autopsy specimens. Like SARS-CoV-1, SARS-CoV-2 infects human cells that express the ACE2 receptor, which is expressed in neurons, leading to the hypothesis that SARS-CoV-2 may invade the CNS $[6,7]$.

Emerging case reports and case series demonstrate evidence of neurological findings in a subset of patients with COVID-19. Two observational series have reported on the prevalence and spectrum of neurological disease in hospitalized patients with COVID-19. A retrospective case series from Wuhan, China analyzed 214 hospitalized patients from three different hospitals with a laboratory confirmed diagnosis of COVID-19. They found 78 of the 214 patients $(36.4 \%)$ had central CNS, peripheral nervous system or skeletal muscle symptoms. The most common central nervous system findings were dizziness $(16.8 \%)$, and headache $(13.1 \%)$. In those with severe COVID-19 disease, $14.8 \%$ displayed encephalopathy [8]. In a case series reported from two centers in France, patients admitted to the hospital because of acute respiratory distress syndrome due to COVID-19 were similarly noted to have high rates of neurological symptoms. Twenty-six of 40 patients were noted to have confusion. Similarly to the patient in this report, that case series found no evidence for SARS-CoV-2 neuroinvasion by PCR of CSF, and EEG findings were non-specific [9].

Isolated case reports have also emerged describing cases of acute encephalopathy associated with COVID19, including a case of COVID-19-associated acute necrotizing encephalopathy, which is typically a postinfectious immune-mediated phenomenon [10]. To date, there has been a single publication reporting possible COVID-19-associated meningoencephalitis (presenting as altered mental status in setting of new onset seizures) in which SARS-CoV-2 was detected in CSF [11]. More recent autopsy studies have detected low levels of SARSCoV-2 RNA in brain tissue in a subset (7 out of 22) of patients who died with COVID-19 [12].

Our findings in this case suggest the possibility that neurological symptoms in COVID-19 may be due to increased neuroinflammation rather than to CNS invasion of virus, and that treatment of neurological complications of COVID-19 may require targeting host-inflammation. We found elevation of MCP-1 in CSF. This proinflammatory chemokine is expressed by numerous cell types within the brain, including neurons, astrocytes, and microglia, resulting in a recruitment of inflammatory infiltrate into the CNS [13]. MCP-1 is elevated in the CSF in other neuroinflammatory and neuroinfectious disorders, including multiple sclerosis, bacterial meningitis and HIV infection [14-16]. CSF elevation of MCP-1 as well as emerging reports of macrophage induced damage in lung and other tissue suggest that specifically attenuating monocyte-incurred damage during COVID-19 may prove beneficial [17]. The findings in this single case should be 
followed up in further cases of seizure and encephalopathy during COVID-19.

In summary, this case report illustrates neurological disease as an initial presentation of COVID-19, in association with elevated inflammatory markers in CSF. The underlying pathophysiology of neurological manifestations of COVID-19 remains incompletely understood and deserves further investigation.

\section{Abbreviations}

CSF: Cerebrospinal fluid; PCR: Polymerase chain reaction; IL: Interleukin; IP10: IFN-y-inducible protein 10; MCP-1: Monocyte Chemoattractant Protein-1; CNS: Central nervous system; EEG: Electroencephalogram; CoVs: Coronavirus

\section{Acknowledgements}

None.

\section{Authors' contributions}

SF and LB designed the study, acquired and interpreted the data, and have agreed both to be personally accountable for the accuracy and integrity of the entire work. LG and JT provided clinical care to the patient, performed literature searches, interpreted the data, and drafted the manuscript. CV, JF, AW, CW, TA, and NG collected, analyzed, and interpreted virological data including sequencing and viral culture. JC, JZ, CT, MS, PP, and SM collected, analyzed, and interpreted cytokine data. AK, SS, ACM, CO, PV, BG, JF, SB, and CDC contributed to study design and conception, patient consent, and data interpretation. All authors reviewed and revised the manuscript and approved the final manuscript.

\section{Funding}

Dr. Farhadian is supported by NIH K23MH118999, the American Federation for Aging Research, the Robert E. Leet and Clara Guthrie Patterson Trust. Dr Ko is supported by NIAID 5 R01 Al121207. This work was supported by the Beatrice Kleinberg Neuwirth Fund. The funding bodies did not have roles in the design of the study and data collection, analysis, and interpretation and did not play any role in writing the manuscript.

\section{Availability of data and materials}

The datasets used and/or analysed during the current study are available from the corresponding author on reasonable request.

\section{Ethics approval and consent to participate}

The patient was consented for participation in the COVID-19 IMPACT biorepository study at Yale, approved by the Yale University Institutional Review Board, HIC\#2000027690.

\section{Consent for publication}

The patient is aware of this case report submission and has provided written consent for this publication.

\section{Competing interests}

The authors report no competing interests

\section{Author details}

'Department of Internal Medicine, Section of Infectious Diseases, Yale School of Medicine, New Haven, CT 06510, USA. ²Department of Neurology, Yale School of Medicine, New Haven, CT 06510, USA. ${ }^{3}$ Department of Internal Medicine, Yale School of Medicine, New Haven, CT 06510, USA. ${ }^{4}$ Department of Epidemiology of Microbial Diseases, Yale School of Public Health, New Haven, CT 06510, USA. ${ }^{5}$ Isoplexis, Branford, CT 06540, USA. ${ }^{6}$ Department of Internal Medicine, Section of Pulmonary, Critical Care, and Sleep Medicine, Yale School of Medicine, New Haven, CT 06510, USA. ${ }^{7}$ Department of Laboratory Medicine, Yale School of Medicine, New Haven, CT 06510, USA.
Received: 1 May 2020 Accepted: 1 June 2020

Published online: 18 June 2020

\section{References}

1. Geleris J, Sun Y, Platt J, Zucker J, Baldwin M, Hripcsak G, et al. Observational study of hydroxychloroquine in hospitalized patients with Covid-19. N Engl J Med. 2020; NEJMoa2012410. https://doi.org/10.1056/NEJMoa2012410. Online ahead of print.

2. Arbour N, Côté G, Lachance C, Tardieu M, Cashman NR, Talbot PJ. Acute and persistent infection of human neural cell lines by human coronavirus OC43. J Virol. 1999:73(4):3338-50.

3. Jacomy $H$, Talbot PJ. Vacuolating encephalitis in mice infected by human coronavirus OC43. Virology. 2003;315(1):20-33.

4. Arabi Y, Harthi A, Hussein J, Bouchama A, Johani S, Hajeer A, et al. Severe neurologic syndrome associated with Middle East respiratory syndrome corona virus (MERS-CoV). Infection. 2015;43(4):495-501.

5. Wu Y, Xu X, Chen Z. Nervous system involvement after infection with COVID-19 and other coronaviruses. Brain Behav Immun. 2020;S08891591(20):30357 Published online March 30, 2020.

6. Netland J, Meyerholz D, Moore S, Cassell M, Perlman S. Severe acute respiratory syndrome coronavirus infection causes neuronal death in the absence of encephalitis in mice transgenic for human ACE2. J Virol. 2008:82(15):7264-75.

7. Xu J, Zhong S, Liu J, Li L, Li Y, Wu X, et al. Detection of severe acute respiratory syndrome coronavirus in the brain: potential role of the chemokine Mig in pathogenesis. Clin Infect Dis. 2005;41(8):1089-96.

8. Mao L, Wang M, Chen S, He Q, Chang J, Hong C, et al. Neurological manifestations of hospitalized patients with COVID-19 in Wuhan, China: a retrospective case series study. JAMA Neurol. 2020;1:e201127.

9. Helms J, Kremer S, Merdji H, Clere-Jehl R, Schenck M, Kummerlen C, et al. Neurologic features in severe SARS-CoV-2 infection. N Engl J Med. 2020; 382(23):2268-70. https://doi.org/10.1056/NEJMc2008597. Epub 2020 Apr 15.

10. Poyiadji N, Shahin G, Noujaim D, Stone M, Patel S, Griffith B. COVID-19-associated acute hemorrhagic necrotizing encephalopathy: $C T$ and MRI features. Radiology. 2020;201187. https://doi.org/10.1148/radiol.2020201187. Online ahead of print.

11. Moriguchi T, Harii N, Goto J, Harada D, Sugawara H, Takamino J, et al. A first case of meningitis/encephalitis associated with SARS-Coronavirus-2. Int J Infect Dis. 2020:94:55-8.

12. Puelles VG, Lutgehetmann M, Lindenmeyer MT, Sperhake JP, Wong MN, Allweiss $\mathrm{L}$, et al. Multiorgan and renal tropism of SARS-CoV-2. N Engl J Med. 2020; NEJMc2011400. https://doi.org/10.1056/NEJMc2011400. Online ahead of print.

13. Yao Y, Tsirka SE. Monocyte chemoattractant protein-1 and the blood-brain barrier. Cell Mol Life Sci. 2014;71:683-97.

14. Weiss JM, Nath A, Major EO, Berman JW. HIV-1 tat induces monocyte chemoattractant protein-1-mediated monocyte transmigration across a model of the human blood-brain barrier and up-regulates CCR5 expression on human monocytes. J Immunol. 1999;163(5):2953-9.

15. Mahad DJ, Ransohoff RM. The role of MCP-1 (CCL2) and CCR2 in multiple sclerosis and experimental autoimmune encephalomyelitis (EAE). Semin Immunol. 2003;15:23-32.

16. Mastroianni CM, Lancella L, Mengoni F, Lichtner M, Santopadre P, D'Agostino C, et al. Chemokine profiles in the cerebrospinal fluid (CSF) during the course of pyogenic and tuberculous meningitis. Clin Exp Immunol. 1998;114:210-4.

17. Liao M, Liu Y, Yuan J, Wen Y, Xu G, Zhao J, et al. Single-cell Landscape of Bronchoalveolar Immune Cells in Patients With COVID-19. Nat Med. 2020. https://doi.org/10.1038/s41591-020-0901-9. Online ahead of print.

\section{Publisher's Note}

Springer Nature remains neutral with regard to jurisdictional claims in published maps and institutional affiliations. 